\title{
Irreversible Anaerobic Photoreactions of Phenylazopyrazolone-Dyes in Solution
}

\author{
J. Zechner and N. Getoff* \\ Institut für Theoretische Chemie und Strahlenchemie der Universität Wien, Austria
}

St. Stoyanov

Chemical Department of Sofia University, Sofia 1126 (Bulgaria)

Z. Naturforsch. 40 a, 37-42 (1985); received November 3, 1984

Dedicated to Prof. Dr. K. Schlögl on the occasion of his $60^{\text {th }}$ anniversary

Irreversible degradation reactions of some 1-phenyl-3-methyl-4-arylazopyrazole-5-ones in deoxygenated solutions were studied by flash and steady state photolysis. In addition to a reversible photoisomerization a reduction of the substrates under investigation to amino compounds was also observed. Degradation quantum yields were found to be lowest in hydrocarbon solvents. They show a pronounced wavelength dependence in all used solvents. Possible reaction mechanisms are discussed.

\section{Introduction}

Irreversible fading of azo-dyestuffs, which are of major commercial interest, is mainly accomplished by photoinduced oxidation and reduction under aerobic resp. anaerobic conditions [1]. The compounds used in the present investigations are derivatives of 1-phenyl-3-methyl-4-arylazo-pyrazol-5-ones (also denoted as 5-methyl-2-phenyl-(2 H-pyrazole3,4-dione-4(Z)-phenylhydrazone); PAP-derivatives). They may exist in one of two possible tautomeric forms, the keto-hydrazone $(\mathrm{H})$ form, or the azoenole form. As a rule in solution they are totally in the $\mathrm{H}$-form, and only in strongly alkaline media $(\mathrm{pH}>11)$ they transform into the anion form (A), common to both, the hydrazone and to the azo-form $[2,3]$. Fluorescence stems exclusively from the $\mathrm{H}$ form [2].

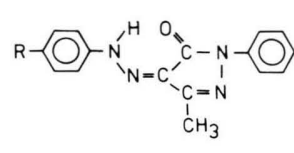

(Ke to-form)

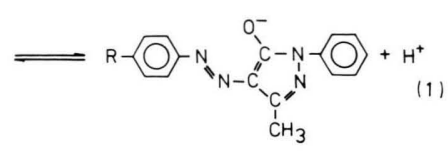

(Enolate-form)
$R=(\mathrm{I})-\mathrm{N}\left(\mathrm{CH}_{3}\right)_{2} ;$ (II) $-\mathrm{NH}_{2} ;$ (III) $-\mathrm{H} ;$ (IV) $-\mathrm{CN} ;(\mathrm{V})-\mathrm{Cl}$

It has been shown [4] that some essential photochemical processes (like photoreduction or photoisomerization) of imino compounds (e.g. hydra-

\footnotetext{
* Reprint requests to Prof. Dr. N. Getoff, Institut für Theoretische Chemie und Strahlenchemie der Universität Wien, Währingererstraße 38, A-1090 Wien/Österreich.
}

zones, Schiff-bases) are in principal similar to those of azo-compounds. In addition to the above mentioned irreversible processes, also photoisomerization reactions around the $\mathrm{N}=\mathrm{N}$ double bond (resp. the $\mathrm{C}=\mathrm{N}$ bond) have been observed, resulting in a reversible photochromic effect [5-8].

In a previous work [9] flash photolysis experiments of phenylazopyrazolone derivatives under anaerobic conditions have been undertaken. It was shown that besides photoisomerization reactions also photoreduction to phenylhydrazyl radicals occurs. This process takes place only when substances are present which can transfer $\mathrm{H}$-atoms to the dyes excited states, e.g. aliphatic alcohols or $\mathrm{N}$-allylthiorea. The spectra of these radicals show a characteristic $\mathrm{pH}$-dependence.

The purpose of the present study was to follow the fate of these hydrazyl radicals and to investigate the formation of final products. Particular respect was to be paid to solvent effects and to the behaviour of both, the hydrazone and the azo-enolate forms.

\section{Experimental}

The synthesis of the studied compounds was accomplished by the standard procedure of diazotization of the corresponding substituted anilines and subsequent coupling in alkaline solution with 1-phenyl-3-methyl-pyrazolone-5 as described elsewhere [10].

Methanol, ethanol and 2-propanol were purified by distillation over 2,4-diphenylhydrazone. Water 
was distilled four times. All other chemicals were reagent grade.

In the flash photolysis experiments the solutions contained $4 \times 10^{-6}$ to $5 \times 10^{-5} \mathrm{~mol} \cdot \mathrm{dm}^{-3}$ substrate and $10 \mathrm{~mol} \cdot \mathrm{dm}^{-3}$ ethanol if not stated otherwise. For deoxygenation the solutions were purged with $\mathrm{O}_{2}$-free argon. The temperature was $25^{\circ} \mathrm{C}$.

The equipment for flash photolysis (pulse length $\leqq$ $7 \mu \mathrm{s}, 20-200 \mathrm{~J}$ ) has been described [11]. Absorption spectra of table compounds were taken either on a Perkin-Elmer Coleman 575 spectrophotometer or on a Specord M 40 (Carl Zeiss, Jena).

In the steady state experiments solutions of $5 \times 10^{-5} \mathrm{~mol} \cdot \mathrm{dm}^{-3}$ substrate were irradiated in $2 \mathrm{~cm}$ quartz cells with a medium pressure mercury lamp (Tungsram HGO $125 \mathrm{~W}$ ). Photodecomposition was usually confined to about $60 \%$ as monitored by means of the substrate absorption maxima at $400 \mathrm{~nm}$. For the analysis with thin layer chromatography (TLC) solutions from several irradiations were collected and concentrated 100 to 1 . TLC was performed with "silicagel without fluorescence indicator" plates (E. Merck, Darmstadt) using benzene acetone $(3: 2)$ as the mobile phase. The individual zones were scratched from the plate and eluted with ethanol in order to take uv spectra.

Quantum yield determinations were carried out at 250 andf $450 \mathrm{~nm}$ using a $450 \mathrm{~W}$ xenon lamp in combination with a high intensity monochromator (Schoeffel Kratos). Light intensities were monitored by means of a photoelectric device (Yellow Springs Instruments Kettering model $65 \mathrm{~A}$ radiometer), which was calibrated by means of chemical actinometry (potassium trioxalatoferrate(III) [12].

\section{Results}

\subsection{Flash photolysis experiments}

Flash photolysis experiments of aqueous $4 \times$ $10^{-6} \mathrm{~mol} \cdot \mathrm{dm}^{-3}$ PAP-derivatives in the presence of $10 \mathrm{~mol} \cdot \mathrm{dm}^{-3}$ methanol showed the formation of transients with two absorption bands $(<350 \mathrm{~nm}$ and $460-470 \mathrm{~nm})$ and a simultaneous degradation of the starting compound $(\lambda=350-450 \mathrm{~nm}$; Figs. 1 and 2). The absorption spectra of both, substrate and transients, are usually partly overlapping. The measured spectra were therefore corrected by means of a computer program.
The results obtained in this way for PAP I and IV in neutral and alkaline solution are shown in Figs. 1 and 2.

Spectral and kinetic data are compiled in Table 1. Because of the simultaneously occurring photoisomerization processes [9] the extinction coefficients $(\varepsilon)$ of the free radicals could not be evaluated

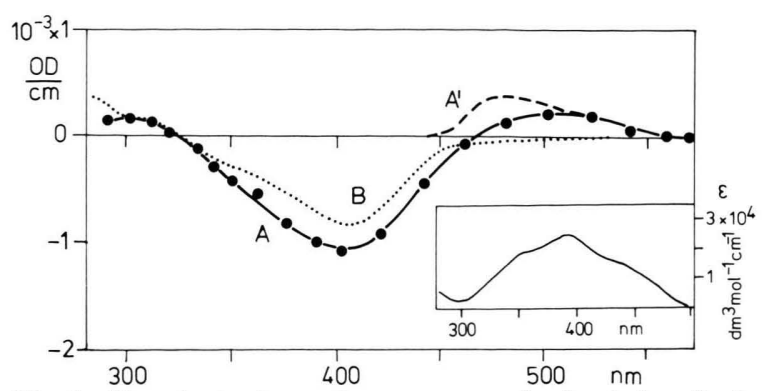

Fig. 1. Transient absorption spectra obtained by flash photolysis (discharge voltage $15 \mathrm{kV}$ ) of deoxygenated solutions of $4 \times 10^{-6} \mathrm{~mol} \cdot \mathrm{dm}^{-3}$ phenylazopyrazolone in $10 \mathrm{~mol} \cdot \mathrm{dm}^{-3}$ aqueous methanol $(\mathrm{pH}=7):$ A) $500 \mu \mathrm{s}$ after flash; $\mathrm{A}^{\prime}$ ) corrected for absorption of the starting compound (see text); B) $3 \mathrm{~s}$ after flash. Insert: UV absorption spectrum of phenylazopyrazolone in $10 \mathrm{~mol} \cdot \mathrm{dm}^{-3}$ aqueous methanol $(\mathrm{pH}=7)$.

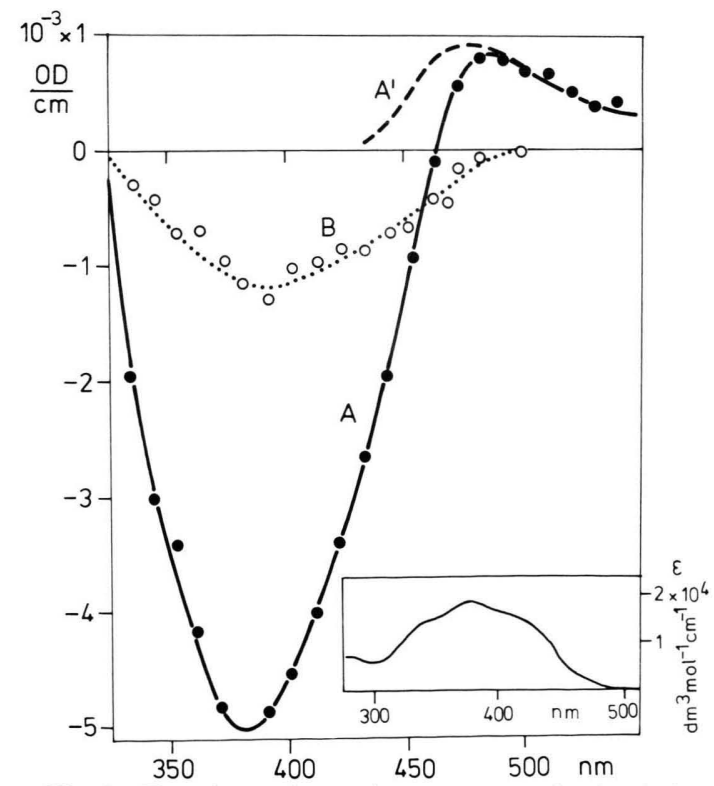

Fig. 2. Transient absorption spectra obtained by flash photolysis (discharge voltage $15 \mathrm{kV}$ ) of deoxygenated solutions of $4 \times 10^{-6} \mathrm{~mol} \cdot \mathrm{dm}^{-3}$ phenylazopyrazolone in $10 \mathrm{~mol} \cdot \mathrm{dm}^{-3}$ aqueous methanol $(\mathrm{pH}=12)$ : A) $200 \mu \mathrm{s}$ after flash; $\mathrm{A}^{\prime}$ ) corected for absorption of the starting compound (see text); B) $3 \mathrm{~s}$ after flash. Insert: UV absorption spectrum of phenylazopyrazolone in $10 \mathrm{~mol} \cdot \mathrm{dm}^{-3}$ aqueous methanol $(\mathrm{pH}=12)$. 
Table 1. Spectral and kinetic data of transients produced by flash photolysis of phenylazopyrazolone derivatives (PAP) in deoxygenated aqueous $10 \mathrm{~mol} \cdot \mathrm{dm}^{-3}$ methanol (discharge voltage $15 \mathrm{kV}$; flash length: $5 \mu \mathrm{s})$.

\begin{tabular}{|c|c|c|c|c|c|c|c|}
\hline \multirow{3}{*}{$\begin{array}{l}\text { PAP } \\
\text { No. }\end{array}$} & \multirow{3}{*}{$\begin{array}{l}\text { Substituent } \\
(\mathrm{X})\end{array}$} & \multicolumn{3}{|c|}{ Kinetics at: } & \multicolumn{3}{|c|}{ Kinetics at: } \\
\hline & & \multirow[b]{2}{*}[\mathrm{nm}]{} & \multicolumn{2}{|l|}{$\mathrm{pH}=7$} & \multirow[b]{2}{*}[\mathrm{nm}]{} & \multicolumn{2}{|c|}{$\mathrm{pH}=12$} \\
\hline & & & $\begin{array}{l}k_{1} \\
{\left[\mathrm{~s}^{-1}\right]}\end{array}$ & $\begin{array}{l}2 \mathrm{k} / \varepsilon \\
{[\mathrm{s} / \mathrm{cm}]}\end{array}$ & & $\begin{array}{l}k_{1} \\
{\left[\mathrm{~s}^{-1}\right]}\end{array}$ & $\begin{array}{l}2 \mathrm{k} / \varepsilon \\
{[\mathrm{s} / \mathrm{cm}]}\end{array}$ \\
\hline $\begin{array}{l}\text { I } \\
\text { II } \\
\text { III } \\
\text { IV } \\
\text { V }\end{array}$ & $\begin{array}{l}-\mathrm{N}\left(\mathrm{CH}_{3}\right)_{2} \\
-\mathrm{NH}_{2} \\
-\mathrm{H} \\
-\mathrm{CN} \\
-\mathrm{Cl}\end{array}$ & $\begin{array}{l}570 \\
560 \\
470 \\
460 \\
460\end{array}$ & $\begin{array}{l}1 \times 10^{3} \\
- \\
- \\
1 \times 10^{3} \\
-\end{array}$ & $\begin{array}{l}8 \times 10^{3} \\
- \\
- \\
4 \times 10^{4} \\
-\end{array}$ & $\begin{array}{l}555 \\
550 \\
475 \\
505 \\
485\end{array}$ & $\begin{array}{l}1 \times 10^{2} \\
5 \times 10^{4} \\
5 \times 10^{4} \\
1 \times 10^{3} \\
-\end{array}$ & $\begin{array}{l}1.1 \times 10^{4} \\
3.9 \times 10^{5} \\
3.5 \times 10^{5} \\
3.0 \times 10^{5} \\
-\end{array}$ \\
\hline
\end{tabular}

by comparison of transient absorption and the bleaching of the starting compound. A rough estimate of the extinction coefficients gives an order of magnitude of $10 \mathrm{dm}^{3} \mathrm{~mol}^{-1} \mathrm{~cm}^{-1}$, both in neutral and alkaline solutions, with higher values for derivatives with electron donating substituents, such as $-\mathrm{NH}_{2}$ and $-\mathrm{N}\left(\mathrm{CH}_{3}\right)_{2}$.

The decay of the transients in many cases appears to be complex. Experiments at various flash intensities allowed us to separate contributions of first and a second order reactions (dimerization or/and disproportionation) (Table 1). As the extinction coefficients are not known exactly, the second order rate constants can only roughly be estimated. The data suggest nearly diffusion controlled dimerization or/ and disproportionation processes.

The absorption of the starting compounds is partly recovered within $\sim 1 \mathrm{~ms}$ in neutral solutions. In alkaline media $(\mathrm{pH}=12)$ two kinetic components for the re-formation can be distinguished: 1) With halflife time $\tau / 2=500 \mu$ s and 2) $\tau / 2=1 \mathrm{~s}$. Thereby $60-70 \%$ of the initial absorption of the substrate are recovered within $3 \mathrm{~s}$. The decay of the fast component is qualitatively in the same time range as that of the transient.

Transients with very similar spectral characteristics as in neutral aqueous solutions were also obtained in pure methanol. In this solvent also a slow kinetic component in the re-formation of the initial absorption (halflife time $\tau / 2 \cong 1 \mathrm{~s}$ ) was observed.

These transients are not formed in cyclohexane or dioxane. In these solvents generally degradation of the substrate and the formation of another type of species could be observed. The absorption maxima of these transients, however, are in all cases situated at too short wavelengths to be measured under our experimental conditions. The bleaching processes are partly reversible; the halflife times for their decay, as well as for the re-formation of the dye are approximately the same, namely: $\tau / 2 \cong 10 \mathrm{~ms}$ in cyclohexane and $\tau / 2 \cong 500 \mu$ s in dioxane. In no case full reversibility was observed. On the other hand, in alcohols and in aqueous solutions the observation of this reversible reaction was not directly possible because of obviously different simultaneous processes. However, contributions to the kinetics of the re-formation of the dye must be assumed.

\subsection{Steady state photolysis}

The spectra of $4^{\prime}$-cyanophenylazopyrazolone(IV) in cyclohexane and of $4^{\prime}$-dimethylaminophenylazopyrazolone(I) in ethanol irradiated at various uvdoses are shown in Figs. 3 and 4. In ethanol (Fig. 4)

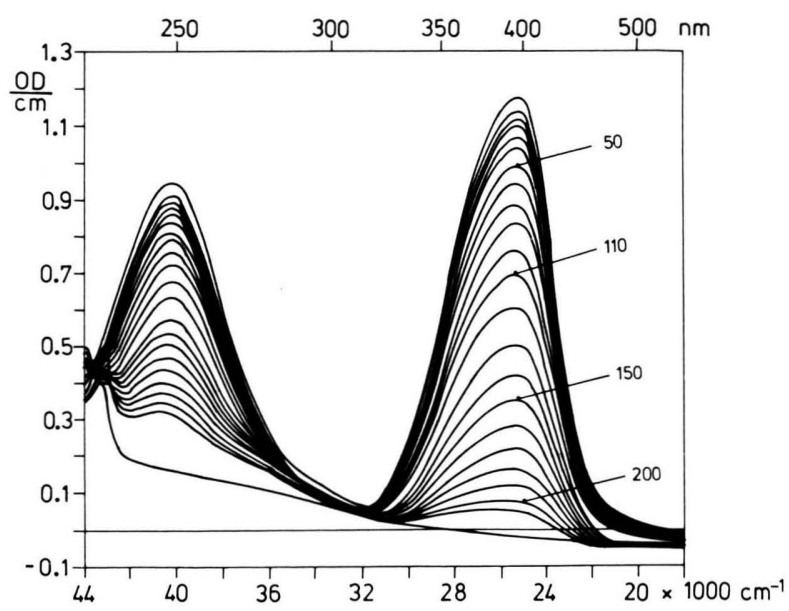

Fig. 3. Spectral changes in the UV-spectra of $4^{\prime}$-cyanophenylazopyrazolone(IV) in cyclohexane. The numbers on the curves give the irradiation time in minutes. 


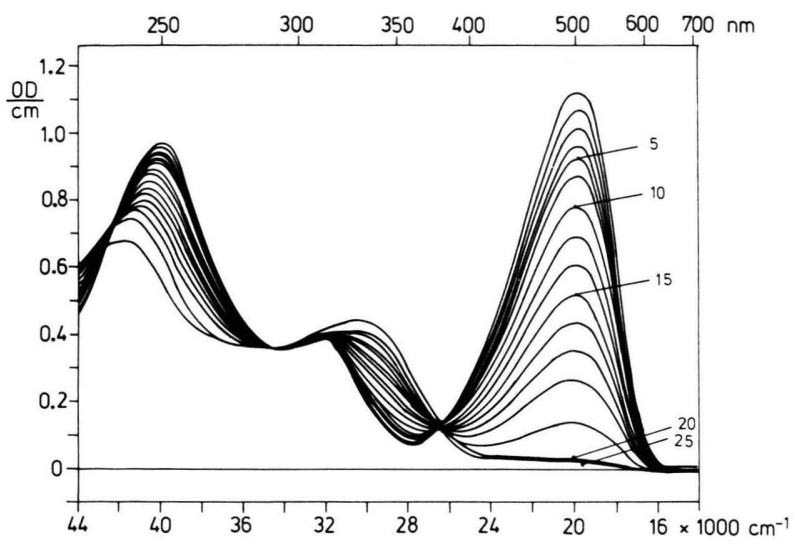

Fig. 4. Spectra changes in the UV-spectra of $4^{\prime}$-dimethylaminophenylazopyrazolone(I) in ethanol. The numbers on the curves give the irradiation time in minutes.

Table 2. Degradation quantum yields $\left(Q_{\mathrm{D}}\right)$ of $4^{\prime}$-dimethylaminophenylazopyrazolone (PAP-I) in various solvents at room temperature.

\begin{tabular}{lll}
\hline Solvent & \multicolumn{2}{l}{$Q_{\mathrm{D}}$ determined at: } \\
\cline { 2 - 3 } & $250 \mathrm{~nm}$ & $450 \mathrm{~nm}$ \\
\hline Cyclohexane & 0.0105 & $1.64 \times 10^{-5}$ \\
Dioxane & 0.01 & - \\
Dioxane+1 m ethanol & 0.0138 & - \\
Dioxane/ethanol = 1:1 & 0.015 & - \\
Ethanol & 0.019 & - \\
Propanol-2 & 0.0436 & $1.55 \times 10^{-5}$ \\
$\mathrm{H}_{2} \mathrm{O} /$ ethanol = 1:1 (pH 7) & 0.0176 & $1 \times 10^{-5}$ \\
$\mathrm{H}_{2} \mathrm{O} /$ ethanol = 1:1(pH 12) & 0.012 & - \\
\hline
\end{tabular}

the existence of isosbestic points around 290 and $390 \mathrm{~nm}$ and the formation of stable absorptions in the near uv-range are observed, whereas in cyclohexane only products are formed which absorb below $300 \mathrm{~nm}$.

The absorption maxima of the products formed from the various substrates in ethanol are for: $\mathrm{R}-\mathrm{H}: 280 \mathrm{~nm}, \mathrm{R}-\mathrm{CN}: 280 \mathrm{~nm}, \mathrm{R}-\mathrm{NH}_{2}: 325 \mathrm{~nm}$ and $\mathrm{R}-\left(\mathrm{CH}_{3}\right)_{2}: 330 \mathrm{~nm}$. They are in good agreement with those of the corresponding 4-substituted anilines.

The degradation quantum yields $\left(Q_{\mathrm{D}}\right)$ for $4^{\prime}$-dimethylaminophenylazopyrazolone (PAP-I) were determined at 250 and $450 \mathrm{~nm}$ in various solvents and are compiled in Table 2 . The pronounced wavelength dependence as well as the solvent effects should be noted.

TLC separation of final products produced in irradiated solutions of PAP-III both in ethanol and in ethanol-water mixtures $(1: 1)$ at $\mathrm{pH}=11$ yielded three main zones:

a) $R_{\mathrm{f}}=0.74$, which was proven to be the unchanged dye both by its $R_{\mathrm{f}}$-value and uv spectra.

b) $R_{\mathrm{f}}=0.27$, identified as aniline by means of $R_{\mathrm{f}}$ and uv-spectra both in alkaline and in acidic medium.

c) $R_{\mathrm{f}}=0.04$; the $R_{\mathrm{f}}$-values and absorption spectrum are identical with those of the photoproduct obtained from aminopyrazolone in ethanolic solution. Its structure was not further investigated.

Formation of azobenzene in substantial amounts could be excluded.

The same products were found both with the hydrazo and with the enolate form.

\section{Discussion}

As mentioned above, the studied compounds exist almost totally in the keto-hydrazo form and only in alkaline solutions in the enolate form. It has been shown that the chromophore of the long wavelength transition is formed by the charge transfer system between the $\mathrm{C}=\mathrm{O}$ group and $\mathrm{R}-\mathrm{Ph}$ [13]. This transition is of $\pi \cdot \pi^{*}$ character $(\varepsilon=2.6 \times$ $10^{4} \mathrm{dm}^{3} \mathrm{~mol}^{-1} \mathrm{~cm}^{-1}$ ).

Two different photoinduced processes are clearly distinguishable:

a) A partially reversible photoprocess, leading to a final product which absorbs at lower wavelengths.

b) The formation of hydrazyl radicals.

Process b) is only observed in alcohols or in the presence of hydrogen donating substance, such as $\mathrm{N}$-allylthiourea [9].

The isomerization process can principally be attributed to a keto-enol tautomery, for which the position of the equilibrium is different in the ground state and in the excited state, or to a geometrical isomerization at the $\mathrm{C}=\mathrm{N}$ bond, namely:

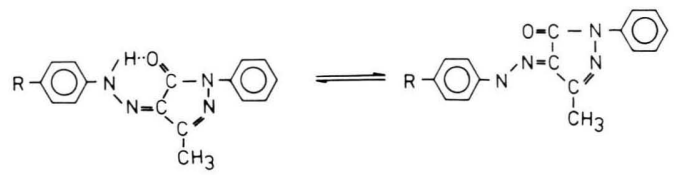

A shift of the keto-enol equilibrium in the $S_{1}$ state is unlikely because of: a) the mirror symmetry between the absorption and emission spectra and 
b) the identity of the emission spectra of the phenylazopyrazolone and a $\mathrm{N}$-methyl substituted derivative in which the keto-hydrazoform is fixed [2]. The photoenolization process cannot be ruled out for the flash photolysis results, if it is assumed that the isomerization proceeds via the triplet state. There is, however, no direct evidence for such a process in our systems.

Similar to azo-compounds syn-anti conformational changes have been observed with a variety of substances with imine structure [5-8]. Except in a few cases $[7,14,15]$ the back reaction appeared to be very fast so that no classical photochromy was observed. The obtained results are not in disagreement with such an isomerization, but as the presented experimental data (Table 1) do not allow to calculate the spectra of the isomerized forms, the final assignment of the transients is still not established. Based on the observed wavelength effects (Table 2) this isomerization reaction seems to originate from the $S_{1}$ state or from a state which is populated from the $S_{1}$. For aromatic Schiff-bases twisting around the azomethine bond in the $S_{1}$ state has been previously observed $[8,16]$, similar to results with azocompounds [17]. Assuming the observed isomerization to originate also from the $S_{1}$ state, the process could account for the high rate of nonradiative decay, which manifests itself in the low degradation quantum yields in the visible region, and probably also in the previously observed low fluorescence quantum yield [2].

As the hydrazyl radicals are only formed in solutions containing reducing substances such as aliphatic alcohols or $\mathrm{N}$-allylthiourea, but not in pure cyclohexane or dioxane [9], the most likely process for their formation is $\mathrm{H}$-abstraction by an excited state from the solvent or from dissolved reducing agents. For a dye reaction in the ground state by photogenerated excited states of impurities or by reactivity intermediates derived from them, as observed for a particular case of imine derivatives [18], there is no indication.

As mentioned above, the quantum yield for degradation of the dyes $\left(Q_{\mathrm{D}}\right)$ in polar as well as in unpolar solvents is almost negligible upon irradiation in the long wavelength band, but is drastically higher at $250 \mathrm{~nm}$ (Table 2). A similar observation was made recently in a study upon reductive cleavage of some azo-dyes $[19,20]$. In this case the effect was explained by the participation of high lying triplet states. However, it has been shown for azodyes that the quantum yield of isomerization is higher upon excitation in the long wavelength band $\left(\pi \cdot \pi^{*}\right)$. It can be assumed that $n \pi^{*}$ states should more easily undergo reduction processes because of their higher polarity. The existence of $\pi^{*}$ states around $300 \mathrm{~nm}$ is suggested by the results of quantumchemical calculations [13]. These states could be populated via higher excited states and enable the reduction processes.

The present results show that further reactions of the hydrazyl radicals ultimately lead to a splitting of the $\mathrm{N}-\mathrm{N}$-bond and finally result in the formation of amines. Two possible mechanisms are suggested for explanation of the hydrazyl radicals decay:

a) Disproportionation of the primary transients:

Reaction 3 :

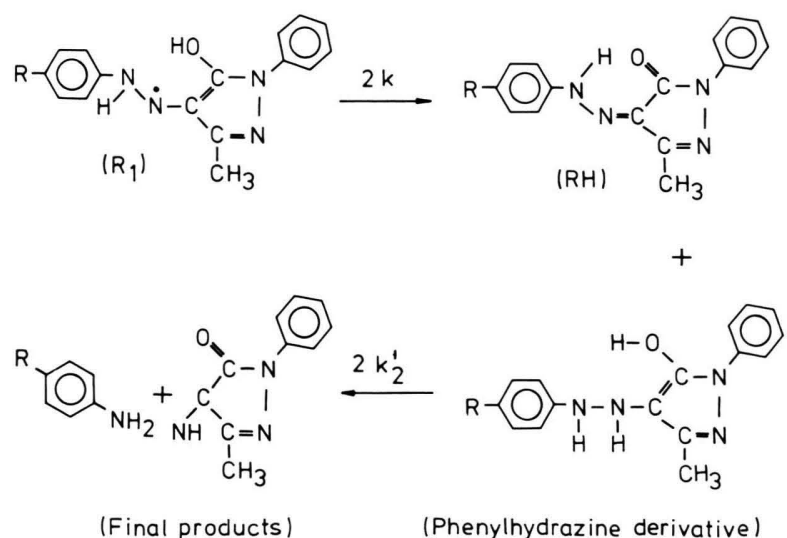

b) Splitting of the primary transients:<smiles>[R]c1ccc([N+](C)(C)N=C2C(C)NN(c3ccccc3)C2O)cc1</smiles><smiles>[R17][R17]C[PH2+]</smiles>

Disproportionation reactions of hydrazyl radicals and subsequently of thus produced hydrazo compounds to amines are known [21-24] and could account for the determined products. On the other 
hand, a mechanism suggesting a splitting of the intermediates has been also observed in ESR studies of related compounds [25].

The obtained kinetic data show a disappearance of the hydrazyl radicals according to a first as well as to a second order process. Disproportionation reactions are naturally favoured under high intensity conditions (flash photolysis), whereas under steady state conditions the "splitting mechanism" should be mainly operative. The relative rates of the two processes obviously reflect substituent and solvent effects. The limited accuracy of the present data, however, prevents a detailed analysis in this respect.

The hydrazine derivatives formed as final products of the disproportionation reaction of the hydrazyl radicals are not definitely observable in the flash experiments because of their short wavelength absorption. They may decompose either by thermal disproportionation [23, 24], yielding substituted aniline and aminopyrazolone, or photo-

[1] J. Griffiths, Chem. Soc. Rev. 1, 481 (1972).

[2] P. Nikolov, F. Fratev, St. Stoyanov, and O. E. Polansky, Z. Naturforsch. 36 a, 191 (1981)

[3] F. A. Snavely and C. H. Yoder, J. Org. Chem. 33, 513 (1968).

[4] A. Padwa, Chem. Revs. 77, 37 (1977).

[5] D. Schulte-Frohlinde, Justus Liebigs Ann. Chem. 622, 47 (1959).

[6] G. Wettermark, M. E. Langmuir, and D. G. Anderson, J. Amer. Chem. Soc. 87, 476 (1965).

[7] A. Maciejewsi and J. Wojtczak, J. Photochem. 21, $351(1983)$

[8] L. L. Costanzo, S. Giuffrida, U. Chiacchio, and G. Condorelli, J. Photochem. 11, 39 (1979).

[9] J. Zechner, G. I. Grabner, N. Getoff, F. Fratev, and St. Stoyanov, J. Photochem. 26, 275 (1984).

[10] G. Schetty, Helv. Chim. Acta 47,921 (1964).

[11] G. Grabner, G. Köhler, J. Zechner, and N. Getoff, Photochem. Photobiol. 26, 449 (1977).

[12] C. G. Hatchard and C. A. Parker, Proc. Roy Soc. London Ser. A, 235, 518 (1956).

[13] D. Simov, St. Stoyanov, and S. Tomov, Ann. de l'Universite de Sofia "Kliment Ohridski", 74, 155 (1979/80).

[14] J. L. Wong and M. F. Zady, J.C.S., Chem. Commun. 1973,694

[15] J. L. Wong and M. F. Zady, J. Org. Chem. 40, 2512 (1975). chemically [26-28] under reformation of hydrazyl radicals. We assume the slow process in the reformation of the dye, as observed especially in alkaline solutions, to reflect essentially the reaction of hydrazine derivatives $(\tau / 2 \cong 5 \mathrm{~s})$, despite the kinetics appear to be complex and most likely contains contributions of some further processes.

\section{Acknowledgements}

The authors appreciate very much the financial support of the Austrian Federal Ministry of Science and Research and of the Bulgarian Academy of Sciences, which made this cooperation possible. Thanks are also expressed to the Fonds zur Förderung der wissenschaftlichen Forschung and to the Ludwig Boltzmann Gesellschaft in Austria for various apparatuses given at our disposal.

We are very grateful for stimulating discussions with the late Doz. Dr. Filip Fratev, Sofia, deceased March 31st 1984, in the early stages of this work.

[16] M. Belletete and G. Durocher, Can. J. Chem. 60, 2332 (1982).

[17] P. Bortolus and S. Monti, J. Phys. Chem. 83, 648 (1979).

[18] A. Padwa, W. Bergmark, and D. Pashayan, J. Amer. Chem. Soc. 91, 2653 (1969).

[19] A. Albini, E. Fasani, and S. Pietra, J. Chem. Soc., Perkin Trans. 2, 1391 (1982).

[20] A. Albini, E. Fasani, and S. Pietra, J. Chem. Soc., Perkin Trans. 2, 1021 (1983); 2, 2491 (1983).

[21] G. Hejkoop and H. C. A. van Beek, Recl. Trav. Chim. Pays Bas 95, 6 (1976).

[22] H. C. A. van Beek, P. M. Heertjes, C. Houtepen, and D. Retzloff, J. Soc. Dyers and Colorists 87,87 (1971).

[23] T. M. Florence, Aust. J. Chem. 18, 619 (1965).

[24] M. Schellenberg and H. Mollett, Helv. Chim. Acta 54, 2431 (1971).

[25] G. Hejkoop and H. C. A. van Beek, Recl. Trav. Chim. Pays Bas 96,83 (1977).

[26] H. Shizuka, H. Kayoiji, and T. Morita, Mol. Photochem. 2, 165 (1970).

[27] S. Hashimoto, J. Sunamoto, and S. Nishitori, Bull. Chem. Soc. Japan 41, 623 (1968).

[28] D. V. Barnthorpe, A. Copper, D. A. Pearce, and J. A. Thomas, J. Chem. Soc. B, 1971, 2077. 